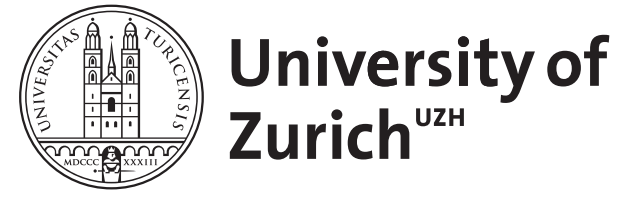

\title{
Computation of the weight distribution of CRC codes
}

\author{
Manganiello, F
}

\begin{abstract}
In this article, we illustrate an algorithm for the computation of the weight distribution of CRC codes. The recursive structure of CRC codes will give us an iterative way to compute the weight distribution of their dual codes starting from some "representative" words. Thanks to MacWilliams' Theorem, the computation of the weight distribution of the dual codes can be easily brought back to that of CRC codes.
\end{abstract}

DOI: https://doi.org/10.1007/s00200-008-0078-9

Posted at the Zurich Open Repository and Archive, University of Zurich ZORA URL: https://doi.org/10.5167/uzh-8520

Journal Article

Accepted Version

Originally published at:

Manganiello, F (2008). Computation of the weight distribution of CRC codes. Applicable Algebra in Engineering, Communication and Computing, 19(4):349-363.

DOI: https://doi.org/10.1007/s00200-008-0078-9 


\title{
COMPUTATION OF THE WEIGHT DISTRIBUTION OF CRC CODES.
}

\author{
FELICE MANGANIELLO
}

\begin{abstract}
In this article, we illustrate an algorithm for the computation of the weight distribution of CRC codes. The recursive structure of CRC codes will give us an iterative way to compute the weight distribution of their dual codes starting from just some "representative" words. Thanks to MacWilliams Theorem, the computation of the weight distribution of dual codes can be easily brought back to that of CRC codes. This algorithm is a good alternative to the standard algorithm that involves listing every word of the code.
\end{abstract}

\section{INTRODUCTION}

Cyclic Redundancy Check (CRC) codes are an important class of error detecting codes. These codes are widely used in computer communication networks because of their easy and fast encoder and decoder implementation and their considerable burst-error detection capability. This properties are provided by the structure of shortened cyclic code. This capability to detect burst-errors is well-studied in [Wic95].

To measure the degree of goodness of error-detecting codes, we have to investigate two properties. The first is the minimum distance of the code. This quantity is the smallest number of bit positions in which any two given words of the code differ. The second is the undetected error probability $\left(P_{u e}\right)$ that measures the probability that an error occurs during transmission that cannot be detected by the decoder. The performance of the code improves when the minimum distance increases or when $P_{u e}$ decreases.

To investigate these two properties, it is important to know the weight distribution of the code. A way to compute this distribution is to list all of the words of the dual code and compute their Hamming weights. The weight distribution of the code is then provided by the Theorem of MacWilliams [MS88].

The structure of CRC codes offers the opportunity to construct an ad-hoc algorithm that has less computational cost; see [CBH93] for a treatment of the binary case.

This work extends the algorithm to CRC codes over any finite field.

The second section of this paper is concerned with preliminary notions. We treat more precisely, but not in detail, CRC codes and properties they have in common with cyclic codes. A good working definition of CRC codes is also given.

The third section deals with the fundamental step of the algorithm. We examine the connection between Linear Recurring Sequences (LRS's) and words of the dual code of a CRC code, explain the need of the choice of the 
best LRS and consider the bijective relation between LRS's and elements of $\mathbb{F}_{q}[x] /(g(x))$, where $g(x)$ is the polynomial generating the code.

We will then turn our interest to the structure of the ring $\mathbb{F}_{q}[x] /(g(x))$. The fourth section shows that it is possible to use the Chinese Remainder Theorem in order to work with quotients rings via powers of irreducible polynomials.

The main task of the fifth section is to find representatives of the $x$-orbits

in the ring $\mathbb{F}_{q}[x] /\left(g(x)^{t}\right)$, where $g(x)$ is an irreducible polynomial. First of all we obtain a decomposition of this ring in an union of sets which are stable under $x$-multiplication. First we go deep into the representation of the multiplicative group of a ring as a product of cyclic groups. A set of generators of these cyclic groups is explicitly shown. Then, we use the preceding results to construct a set of representatives of every possible $x$ orbit of the ring.

\section{Preliminaries}

In the introduction, we stated that CRC codes are extensively used nowadays. Despite that in literature there is differing definitions of this code. This is raised to the different ideas about their utilize. Now we will give a definition of this codes from [Ros01] to then bring back us to the more operational one.

Definition 1. Let $g(x) \in \mathbb{F}_{q}[x]$ be a monic polynomial over the finite field $\mathbb{F}_{q}$ of characteristic $p$. Let us consider the encoding map

$$
\begin{aligned}
\phi: F_{q}[x] & \rightarrow F_{q}[x] \\
m(x) & \mapsto c(x)=m(x) g(x) .
\end{aligned}
$$

A Cyclic Redundancy Check $(C R C)$ code is then the ideal $(g(x))=\operatorname{im} \phi$.

This definition give the basic property of CRC codes, i.e. the fact that they are generated by a generator polynomial $g(x)$.

Such a definition of CRC codes is appropriate from a theoretical point of view, but in application this definition is not enough. The resulting code is not observable, see [Ros01].

One way to correct this problem in to predetermine the length of the message. This allows the receiver to test for code membership by long division. If $c(x)$ is the received word, compute

$$
c(x)=\tilde{m}(x) g(x)+r(x) .
$$

If $r(x)=0$, then the receiver can conclude that $\tilde{m}(x)$ is the transmitted message $m(x)$. Otherwise a retransmission will be requested.

We thus arrive at a better working definition of CRC codes.

Definition 2. Let $n, r \in \mathbb{N}$ with $n>r>0$. Let $q \in \mathbb{N}$ be some power of a prime number $p$ and $g(x) \in \mathbb{F}_{q}[x]$ a monic polynomial such that $\operatorname{deg} g(x)=r$ and $g(0) \neq 0$.

$A(n, n-r) C R C$ code $C$ is the set

$$
C=\left\{c(x) \in \mathbb{F}_{q}[x] \mid c(x)=g(x) m(x), \operatorname{deg} m(x)<n-r\right\} .
$$


Such a set has the structure of a linear code. We note that a CRC code is a cyclic code if and only if the generator polynomial $g(x)$ divides $x^{n}-1$.

From this representation, it is easy to deduce that CRC codes are shortened cyclic codes. In fact, a basis of a CRC code can be formed by $x$ multiplications of the generator polynomial.

As previously stated, the Theorem of MacWilliams give us the possibility to switch our interest to the weight distribution of the dual code.

The dual code of a CRC code has an interesting structure. Given a polynomial $g(x)$ over $\mathbb{F}_{q}$, the dual code of a CRC code of any length generated by $g(x)$ is isomorphic to the ring $\mathbb{F}_{q}[x] /(g(x))$.

Via some easy steps which come from the theory of dual codes, it is easy to deduce the following property of dual codewords:

Proposition 3. Let $C \in \mathbb{F}_{q}^{n}$ be a CRC code of and

$$
g(x)=g_{0}+g_{1} x+\cdots+g_{r-1} x^{r-1}+x^{r}
$$

its generator polynomial. Then $c=\left(c_{0}, \ldots, c_{n-1}\right)$ is an element of the dual code $C^{\perp}$ if and only if its components satisfy the relation

$$
c_{i}=-g_{0} c_{i-r}-\cdots-g_{r-1} c_{i-1}, \quad i=r, \ldots, n .
$$

Notations. In this work, we will use the following notations:

- $p$ will be the prime number that is the characteristic of the $\operatorname{ring} \mathbb{F}_{q}$; then $q$ is a power of $p$, i.e. $q=p^{\delta}$ for some $\delta \in \mathbb{N}_{+}$;

- $n \in \mathbb{N}_{+}$will be the length of the CRC code;

- $g(x) \in \mathbb{F}_{q}[x]$ will be the monic generator polynomial of a CRC code, with $g(0) \neq 0, \operatorname{deg} g(x)=r$ and $0<r<n$;

- $g(x)=\prod_{i=l}^{m} g_{l}(x)^{e_{l}}$ will be the irreducible decomposition of $g(x)$;

- $u$ will be an element of the ring $\mathbb{F}_{q}[x] /(g(x))$ and $u(x)$ the representative of lowest degree of $u$ in $\mathbb{F}_{q}[x]$;

- $\mathcal{R}_{g}^{q}$ will be the ring $\mathbb{F}_{q}[x] /(g(x))$ and $\mathcal{R}_{g^{t}}^{q}$ the ring $\mathbb{F}_{q}[x] /\left(g(x)^{t}\right)$;

- $M_{g}^{q}$ will be the multiplicative group of $\mathcal{R}_{g}^{q}$, i.e. $\left(\mathbb{F}_{q}[x] /(g(x))\right)^{*}$, and $M_{g^{t}}^{q}$ the multiplicative group of the ring $\mathcal{R}_{g^{t}}^{q}$.

\section{Quotient Ring by a Primitive Polynomial and Fundamental Step of the Algorithm}

In this section the fundamental step of the algorithm will be illustrated. We will use algebraic objects such as linear recurring sequences (LRS's) [LN93] and polynomials over finite fields.

The next theorem recalls the part of Kronecker's Theorem [Kro81] which is the most interesting for our purpose.

Theorem 4. Let $u(x) \in \mathbb{F}_{q}[x]$ be a polynomial with $\operatorname{deg} u(x)<\operatorname{deg} g(x)$. Then there exists exactly one sequence $\left(c_{i}\right)_{i \in \mathbb{N}} \subset \mathbb{F}_{q}^{\mathbb{N}}$ such that

$$
\frac{u(x)}{g(x)}=\sum_{i=0}^{\infty} \frac{c_{i}}{x^{i+1}}=: c(1 / x) .
$$

Moreover the sequence $\left(c_{i}\right)_{i \in \mathbb{N}}$ satisfies the linear relation

$$
c_{i}=-g_{0} c_{i-r}-\cdots-g_{r-1} c_{i-1}, \quad i \geq r .
$$


As an immediate application one obtains the following corollary.

Corollary 5. There exists a bijection between the ring $\mathcal{R}_{g}^{q}$ and the set of all $L R S$ with characteristic polynomial $g(x)$.

From the preliminaries stated in the previous section and from Corollary 5 , it follows that there is a bijection between the set of LRS's with characteristic polynomial $g(x)$ and the dual code of any CRC code whose generator polynomial is $g(x)$. In the following theorem, we make this bijection explicit.

Theorem 6. Let $L_{g}$ be the set of LRS's over $\mathbb{F}_{q}$ with characteristic polynomial $g(x)$. Let $C$ be an $(n, n-r) C R C$ code over $\mathbb{F}_{q}$ whose generator polynomial is $g(x)$, and $C^{\perp}$ its dual code. Then the following relation

$$
\begin{aligned}
\psi: L_{g} & \rightarrow C^{\perp} \\
\left(c_{i}\right)_{i \in \mathbb{N}} & \mapsto\left(c_{0}, \ldots, c_{n-1}\right)
\end{aligned}
$$

is bijective.

This relation allows one to work with LRS's instead of with words of the dual code. Now we want to represent any word of the dual code through some LRS. We want to use the minimum possible number of LRS's to representing the dual code. The next lemma will give us the chance to take only some of the LRS's of $L_{g}$ in representing the code $C^{\perp}$ :

Lemma 7. Let $C \subset \mathbb{F}_{q}^{n}$ be a $C R C$ code with generator polynomial $g(x)$, and $\left(c_{i}\right)_{i \in \mathbb{N}} \subset \mathbb{F}_{q}^{\mathbb{N}}$ a LRS whose characteristic polynomial is $g(x)$. Then

$$
\left(c_{k}, \ldots, c_{k+n-1}\right) \in C^{\perp} \quad \forall k \in \mathbb{N}
$$

This Lemma gives us a way to "extract" words of the dual code using only a LRS and the length of the code.

We will now obtain the best way to construct a LRS. We will use the lowest degree representative element of a class of $\mathcal{R}_{g}^{q}$ and divide it by the monic polynomial $g(x)$.

Let now $u(x) \in F_{q}[x]$ be a polynomial satisfying the hypothesis of Theorem 4. A method for obtaining the related LRS is explained in [CBH93]. The method follows from the relation

$$
\frac{u(x)}{g(x)}=\frac{u_{r-1}}{x}+\frac{u^{\prime}(x)}{x g(x)},
$$

where $u_{r-1}$ is the coefficient of the $(p-1)$-th degree term of the polynomial $u(x)$ and $u^{\prime}(x)=x u(x)-u_{r-1} g(x) \equiv x u(x)(\bmod g(x))$. It is trivial to see that the polynomial $u^{\prime}(x)$ satisfies the hypothesis of Theorem 4 as well. Relation (2) can be iterated, and the resulting sequence of coefficients $u_{r-1}$ is a LRS.

An interesting remark is that LRS can be easily constructed by using a Linear Feedback Shift Register (LFSR) with generator polynomial $g(x)$. The next subsection is devoted to the fundamental step of the algorithm.

3.1. The Fundamental Step. Let us now consider a LRS $\left(c_{i}\right)_{i \in \mathbb{N}}$ with characteristic polynomial $g(x)$. We will make the way to extract words of the dual code of a $(n, n-r)$ CRC code explicit. The following figure depicts the idea of algorithm; this scheme follows from Lemma 7. 


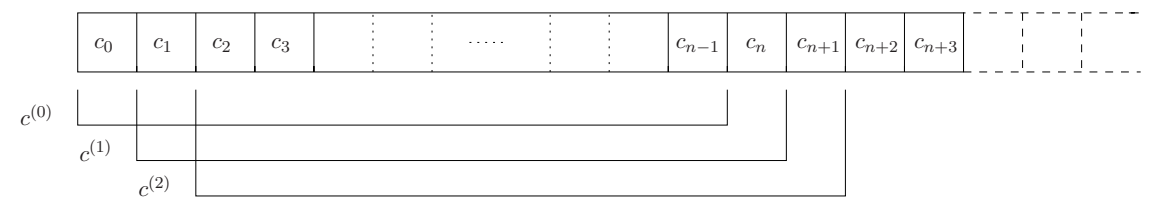

In the figure above, $c^{(k)} \subset \mathbb{F}_{q}^{n}$ denotes the $k$-th word of the dual code extracted from the above sequence.

The figure leads directly to relations between the weight distribution of the words thus extracted:

Remark. (Weight relations between words)

- if $c_{k-1} \neq 0$ and $c_{k+n-1}=0$, then $w t\left(c^{(k)}\right)=w t\left(c^{(k-1)}\right)-1$;

- if $c_{k-1}=0$ and $c_{k+n-1} \neq 0$, then $w t\left(c^{(k)}\right)=w t\left(c^{(k-1)}\right)+1$;

- $w t\left(c^{(k)}\right)=w t\left(c^{(k-1)}\right)$ otherwise.

This remark will be very useful in decreasing the computational cost of the algorithm. In such a way, once the weight of the first word extracted from a LRS $\left(c_{i}\right)_{i \in \mathbb{N}}$ has been computed, the weights of the following words can be easily determined. This procedure has a minimal computational cost, cause the operations of addition or subtraction are constant time complexity operations.

In [CBH93], a way to compute these weights from the same two LFSR is also explained. The second LFSR has to be shifted $n$ times. The first LFSR will give the new input component (bit) of the constructed word and the second one the leaving one.

3.2. Relation Between LRS's and Words of $C^{\perp}$. Now we are able to extract words of the dual code from LRS's, but some questions are still unanswered. What is a minimal set of LRS's sufficient to determine the weight distribution? How can we be sure that we are not considering the same word more than once?

We define next sets:

Definition 8. Let $u(x) \in \mathbb{F}_{q}[x]$ with $\operatorname{deg} u(x)<\operatorname{deg} g(x)$, and $\left(c_{i}\right)_{i \in \mathbb{N}} \subset \mathbb{F}_{q}^{\mathbb{N}}$ be the LRS related to $u(x)$ (see Theorem 4). We denote with $C_{u}^{\perp} \subset C^{\perp}$ the set of all words of the dual code of a CRC code extracted from $\left(c_{i}\right)_{i \in \mathbb{N}}$.

The next Lemma states a bound on the number of different words that can be extracted from a fixed LRS.

Lemma 9. Let $u(x) \in \mathbb{F}_{q}[x]$ such that $\operatorname{deg} u(x)<\operatorname{deg} g(x)$. The cardinality of $C_{u}^{\perp}$ is

$$
\left|C_{u}^{\perp}\right|=\operatorname{ord}\left(\frac{g(x)}{\operatorname{gcd}(g(x), u(x))}\right) .
$$

The proof of Lemma follows directly from the relation between the number of words that can be extracted and the period of $\left(c_{i}\right)_{i \in \mathbb{N}}$ and the definition of the order of a polynomial. Details can be found in [CBH93].

Definition 10. Let $u(x) \in \mathbb{F}_{q}[x]$, the order of $u(x)$ is the least natural number $o_{u}$ such that $u(x)$ divides $x^{o_{u}}-1$. 
3.3. $x$-orbits, LRS and Words of $C^{\perp}$. In order to continue we need another algebraic structure, i.e. the $x$-orbits of the ring $\mathcal{R}_{g}^{q}$. As we have already remarked, the $g(x)$ and $x$ are relatively prime. Let $(\mathrm{x})$ denote the cyclic subgroup of the multiplicative group $M_{g}^{q}$ generated by $x$, i.e.

$$
(\mathrm{x}):=\left\{x^{k} \in M_{g}^{q} \mid k \in \mathbb{N}\right\} .
$$

Definition 11. The $x$-orbits are the sets resulting from the action of the cyclic group (x) on the ring $\mathcal{R}_{g}^{q}$. For $u$ an element of $\mathcal{R}_{g}^{q}$, we denote with $\mathfrak{C}_{u}^{\perp}$ the $x$-orbit of $u$.

It is well known that $x$-orbits can be considered as equivalence classes of the ring $\mathcal{R}_{g}^{q}$.

The next lemma gives an explicit relation between the $x$-orbits of two distinct elements of $\mathcal{R}_{g}^{q}$ and the respectively generated LRS's. The lemma will work with sets of words $C_{u}^{\perp}$, i.e. words extracted from a LRS.

Lemma 12. Let $u_{1}, u_{2}$ be two distinct elements of $\mathcal{R}_{g}^{q}$. Then the following relation

holds.

$$
u_{2} \in \mathfrak{C}_{u_{1}}^{\perp} \Longleftrightarrow C_{u_{1}}^{\perp}=C_{u_{2}}^{\perp}
$$

Proof. The proof consists of two parts.

$(\Rightarrow)$ By definition $u_{2}$ belongs to $\mathfrak{C}_{u_{1}}^{\perp}$ if and only if there exists $j \in \mathbb{N}$ such that

$$
u_{2}(x) \equiv x^{j} u_{1}(x) \quad(\bmod g(x)) .
$$

Hence the LRS constructed from the polynomial $u_{2}(x)$ is the same as the LRS obtained by shifting that of the first polynomial $j$ times, so that $C_{u_{2}}^{\perp} \subset C_{u_{1}}^{\perp}$.

The cardinality of the two sets is the same, as

$$
\begin{aligned}
\left|C_{u_{2}}^{\perp}\right| & =\operatorname{ord}\left(\frac{g(x)}{\operatorname{gcd}\left(g(x), u_{2}(x)\right)}\right)=\operatorname{ord}\left(\frac{g(x)}{\operatorname{gcd}\left(g(x), x^{j} u_{1}(x)\right)}\right)= \\
& =\operatorname{ord}\left(\frac{g(x)}{\operatorname{gcd}\left(g(x), u_{1}(x)\right)}\right)=\left|C_{u_{1}}^{\perp}\right|
\end{aligned}
$$

since $\operatorname{gcd}(x, g(x))=1$. This implies $C_{u_{2}}^{\perp}=C_{u_{1}}^{\perp}$.

$(\Leftarrow)$ Let us suppose that there is no $j \in \mathbb{N}$ such that

$$
u_{2}(x) \equiv x^{j} u_{1}(x) \quad(\bmod g(x)) .
$$

This implies that in the development of relation (2) beginning with $u_{1}(x)$, the polynomial $u_{2}(x)$ cannot be found in the right-hand side of the relation (2). Hence the LRS related to the second polynomial cannot be obtained as a shift of the LRS related to the first one. This implies that the sets $C_{u_{1}}^{\perp}$ and $C_{u_{2}}^{\perp}$ are different.

The proof is complete.

Previously, in Section 2, we stated the bijective relation between the dual code of a CRC code and the ring $\mathcal{R}_{g}^{q}$. It follows from the previous lemma that the dual code can be constructed by taking the union of disjoint sets that are related to the $x$-orbits of the ring $\mathcal{R}_{g}^{q}$. These orbits are also related to LRS's. Our goal is to find a representative of each $x$-orbit. Thereafter using 
the fundamental step we will be able to compute the weight distribution of the dual code.

\section{Application of the Chinese Remainder Theorem}

We want now to obtain a representation of the structure of the ring $\mathcal{R}_{g}^{q}$ that will be useful for our particular algorithm. We will look for a decomposition of the ring into $x$-orbits.

From the Chinese Remainder Theorem we know that

$$
\mathcal{R}_{g}^{q} \approx \prod_{l=1}^{m} \mathcal{R}_{g_{l} e_{l}}^{q},
$$

where $g(x)=\prod_{l=1}^{m} g_{l}(x)^{e_{l}}$ is the irreducible factor decomposition.

Let us write the isomorphism explicitly in our case. The following theorem is claimed implicitly in [CBH93].

Theorem 13. Let $g(x) \in \mathbb{F}_{q}[x]$ be a monic polynomial such that $g(0) \neq 0$, and let us consider its irreducible decomposition.

The map

$$
\phi: \mathcal{R}_{g}^{q} \rightarrow \prod_{l=1}^{m} \mathcal{R}_{g_{l} e_{l}}^{q}
$$

given by $\phi(u)=\left(u_{1}, \ldots, u_{m}\right)$ with

$$
u_{l}(x) \equiv u(x) \quad\left(\bmod g_{l}(x)^{e_{l}}\right)
$$

is an isomorphism with inverse

$$
\phi^{-1}\left(u_{1}, \ldots, u_{m}\right)=\sum_{l=1}^{m} u_{l}(x) v_{l}(x) \frac{g(x)}{g_{l}(x)^{e_{l}}} \quad(\bmod g(x)),
$$

where $v_{l}(x)$ is the multiplicative inverse of $g(x) / g_{l}(x)^{e_{l}}$ in $\mathcal{R}_{g_{l} e_{l}}^{q}$.

Thanks to this theorem we can begin our study in the case of quotient rings of powers of irreducible polynomials.

Let $u_{l}$ be an element of the ring $\mathcal{R}_{g_{l} e_{l}}^{q}$; the result of the action of $x^{k_{l}}$ on $u_{l} \in \mathcal{R}_{g_{l} e_{l}}^{q}$ will be denoted by $u_{l}^{\left(k_{l}\right)}$, i.e. $u_{l}^{\left(k_{l}\right)} \equiv x^{k_{l}} u_{l}\left(\bmod g_{l}(x)^{e_{l}}\right)$. From the paper [CBH93] and some calculations, one obtains the next theorem.

Theorem 14. Let $g(x) \in \mathbb{F}_{q}[x]$ be monic with $g(0) \neq 0$ and $g(x)=\prod_{l=1}^{m} g_{l}(x)^{e_{l}}$ be its irreducible decomposition. Let also $\mathfrak{C}_{u_{l}}^{\perp}$ be the x-orbits of $\mathcal{R}_{g_{l} e_{l}}^{q_{l}}$ for $l=1, \ldots, m$, with cardinality $d_{l}$ and representative $u_{l}$ respectively.

It follows that representatives of any $x$-orbits of the ring $\mathcal{R}_{g}^{q}$ are

$$
\left(u_{1}, u_{2}^{\left(k_{2}\right)}, \ldots, u_{m}^{\left(k_{m}\right)}\right) \in \prod_{l=1}^{m} \mathcal{R}_{g_{l} e_{l}}^{q}
$$

for $0 \leq k_{l}<\mathcal{K}_{l}$, for $l=2, \ldots, m$ and

$$
\mathcal{K}_{l}=\operatorname{gcd}\left(d_{l}, \operatorname{lcm}\left(d_{1}, \ldots, d_{l-1}\right)\right) .
$$

A proof of the previous theorem can be done by induction using the paragraph titled Action of a Cyclic Group on a Cartesian Product of [CBH93], where the authors analyze the case of the cartesian product of two sets. 


\section{Decomposition of $\mathcal{R}_{g^{t}}^{q}$ INTO $x$-ORBIts}

Let $t$ be a natural number and let us consider an irreducible polynomial $g(x) \in \mathbb{F}_{q}[x]$ of degree $r$. The following representation of the elements of $\mathcal{R}_{g^{t}}^{q}$ is also valid and will be useful for our work.

Lemma 15. Every $f \in \mathcal{R}_{g^{t}}^{q}$ can be represented in an unique way as

$$
f=\left[\sum_{l=0}^{t-1} f_{l}(x) g(x)^{l}\right]
$$

where $f_{l}(x) \in \mathbb{F}_{q}[x]$ and $\operatorname{deg} f_{l}(x)<\operatorname{deg} g(x)$.

This representation of an element of $\mathcal{R}_{g^{t}}^{q}$ follows directly from the representation of any representative of the class in $g(x)$ base.

The next step is to investigate subsets of the ring $\mathcal{R}_{g^{t}}^{q}$ which are closed under $x$-multiplication. The following theorem gives information about such subsets.

Theorem 16. Let $u(x) \in \mathbb{F}_{q}[x]$ be the representative of minimal degree of a class $u \in \mathcal{R}_{g^{t}}^{q}$, and let $s$ be the natural number

$$
s:=\max \left\{i \in \mathbb{N}\left|g(x)^{i}\right| u(x)\right\} .
$$

If we denote by $\bar{u}(x) \in \mathbb{F}_{q}[x]$ the polynomial obtained by dividing $u(x)$ by $g(x)^{s}$, then the following relation holds:

$$
u^{\prime} \in \mathfrak{C}_{u}^{\perp} \subset \mathcal{R}_{g^{t}}^{q} \Longleftrightarrow\left\{\begin{array}{l}
\max \left\{i \in \mathbb{N}\left|g(x)^{i}\right| u^{\prime}(x)\right\}=s, \\
{\left[u^{\prime}(x) / g(x)^{s}\right] \in \mathfrak{C}_{\bar{u}}^{\perp} \subset \mathcal{R}_{g^{t-s}}^{q} .}
\end{array}\right.
$$

The proof of this theorem is an easy computation.

Remark. From the previous theorem, two remarks can be extracted:

(1) give an $x$-orbit $\mathfrak{C}_{u}^{\perp}$, the maximal power of $g(x)$ that divides an element of the orbit does not depend of the choice of the element;

(2) the choice of the best representative of $x$-orbits can be limited to the set of elements of $\mathcal{R}_{g^{t}}^{q}$ whose representatives in $\mathbb{F}_{q}[x]$ are coprime with $g(x)$.

The next corollary follows from Lemma 15 and the previous theorem.

Corollary 17. The ring $\mathcal{R}_{g^{t}}^{q}$ can be decomposed as follows:

$$
\mathcal{R}_{g^{t}}^{q}=\{0\} \cup \bigsqcup_{l=1}^{t-1} g(x)^{l} \cdot M_{g^{t-l}}^{q}
$$

where the sets

$$
g(x)^{l} \cdot M_{g^{t-l}}^{q}=\left\{u \in \mathcal{R}_{g^{t}}^{q} \mid u=\left[g(x)^{l} \bar{u}(x)\right], \bar{u} \in M_{g^{t-l}}\right\}
$$

are stable under $x$-multiplication. 
5.1. Characterization of elements of $M_{g^{l}}^{q}$. Let us initially give a corollary of Lemma 15.

Corollary 18. The element $f \in \mathcal{R}_{g^{t}}^{q}$ is invertible if and only if, in the representation given in Lemma 15, $f_{0}(x) \neq 0$.

The group $M_{g^{l}}^{q}$ is the multiplicative group of the ring $\mathcal{R}_{g^{l}}^{q}$ and is finite. By the theory of finitely generated abelian groups, $M_{g^{l}}^{q}$ can be expressed as a product of cyclic groups. Let us investigate this structure more precisely.

Let us distinguish between two cases: $l=1$ and $l \geq 2$. The first case should also be split into two parts: either $g(x)$ is primitive, or it is not. In both cases, the ring $\mathcal{R}_{q}^{q}$ is also a finite field, hence its multiplicative group is cyclic. The difference between the primitive and the non-primitive case lies in the choice of the generator element. If $g(x)$ is a primitive polynomial, i.e. if $\operatorname{ord}(g(x))=q^{r}-1$, where $\operatorname{deg} g(x)=r$, then $x \in M_{g}^{q}$ is a good choice of generator. Otherwise, $x$ is not a generator anymore. We will denote by $h \in M_{g}^{q}$ a generator of the group.

Let us now consider the case $l \geq 2$.

Theorem 19. The order of the group $M_{g^{l}}^{q}$ is $\left(q^{r}-1\right) q^{(l-1) r}$. Moreover

$$
M_{g^{l}}^{q} \approx M_{g}^{q} \times S_{p},
$$

where $S_{p}$ is the p-Sylow subgroup of $M_{g^{l}}^{q}$.

The group $M_{g}^{q}$ has already been analyzed; we now study the structure of the $p$-Sylow subgroup.

Theorem 20. Let $f \in M_{g^{l}}^{q}$. The multiplicative order of $f$ is a power of $p$ if and only if there exists a polynomial $m(x) \in \mathbb{F}_{q}[x]$ such that

$$
f=[1+m(x) g(x)] .
$$

Proof. Let $f(x) \in \mathbb{F}_{q}[x]$ be the representative of lowest degree of $f$, where $f$ is an element whose order is a power of $p$. There exists a unique way to write

$$
f(x)=f_{0}(x)+f^{(1)}(x) g(x)
$$

with $\operatorname{deg} f_{0}(x)<\operatorname{deg} g(x)$.

Let $k \in \mathbb{N}_{+}$be such that $p^{k}>l$. Then

$$
f(x)^{p^{k}}=\left(f_{0}(x)+f^{(1)}(x) g(x)\right)^{p^{k}}=f_{0}(x)^{p^{k}}+\left(f^{(1)}(x) g(x)\right)^{p^{k}} .
$$

In $\mathcal{R}_{g^{l}}^{q}$, this relation reduces to $f^{p^{k}}=\left[f_{0}(x)^{p^{k}}\right]$, and $f_{0}(x)^{p^{k}}$ is the lowestdegree representative of the class. From the equality criteria between polynomials, we see that $f_{0}(x)=1$.

Vice-versa, let $m(x) \in \mathbb{F}_{q}[x]$ be a polynomial such that

$$
f(x)=1+m(x) g(x) .
$$

Let $k \in \mathbb{N}_{+}$be such that $p^{k}>l$; then

$$
f(x)^{p^{k}}=(1+m(x) g(x))^{p^{k}}=1+(m(x) g(x))^{p^{k}},
$$

and this represents the identity in $\mathcal{R}_{g^{l}}^{q}$. 
Remark. From now on we will denote by $\alpha$ an element algebraic over $\mathbb{F}_{p}$ of degree $\delta$. Recall that $\delta$ is such that $q=p^{\delta}$. Therefore, we obtain $\mathbb{F}_{q} \approx \mathbb{F}_{p}[\alpha]$.

Another notation that we will use extensively is the following:

$$
a_{i, j, k}(x)=1+\alpha^{i} x^{j} g(x)^{k} \in S_{p},
$$

where $0 \leq i<\delta, 0 \leq j<r$ and $1 \leq k<l$. We are now able to state the theorem that specifies the decomposition of the $p$-Sylow subgroup into a product of cyclic groups.

Theorem 21. Let $S_{p}$ be the $p$-Sylow subgroup of $M_{g^{l}}^{q}$. The following isomorphism holds:

$$
S_{p} \approx \prod_{i, j, k}\left(a_{i, j, k}(x)\right),
$$

where $\left(a_{i, j, k}(x)\right) \subset S_{p}$ is the cyclic group generated by $a_{i, j, k}(x)$ and the parameter $k$ satisfies the condition $p \nmid k$.

The proof of this theorem follows from the next lemma.

Lemma 22. For any polynomial

$$
f(x)=1+f_{h}(x) g(x)^{h}+m(x) g(x)^{h+1} \in \mathbb{F}_{q}[x]
$$

with $h \in \mathbb{N}_{+}$and $\operatorname{deg} f_{h}(x)<\operatorname{deg} g(x)$, there exist numbers

$$
c_{(i, j)} \in\{0,1, \ldots, p-1\}
$$

such that

$$
\prod_{i, j} a_{i, j, h}(x)^{c_{(i, j)}} \equiv f(x) \quad\left(\bmod g(x)^{h+1}\right)
$$

for every $0 \leq i<\delta$ and $0 \leq j<r$.

Proof. The proof will be split into two parts: either $p \nmid h$, or $p \mid h$.

Let us first suppose that $p \nmid h$. The polynomial $f_{h}(x)$ can be written as

$$
f_{h}(x)=\sum_{i, j} c_{(i j)} \alpha^{i} x^{j}
$$

with $c_{(i j)} \in \mathbb{F}_{p}$. It follows that

$$
\begin{aligned}
\prod_{i, j} a_{i, j, h}(x)^{c_{(i j)}} & =1+\sum_{i, j} c_{(i j)} \alpha^{i} x^{j} g(x)^{h}+m^{\prime}(x) g(x)^{h+1} \equiv \\
& \equiv f(x) \quad\left(\bmod g(x)^{h+1}\right) .
\end{aligned}
$$

Otherwise, if $p \mid h$ then $h=p h^{\prime}$. The polynomial $g(x)$ is irreducible, thus the field $\mathcal{R}_{g}^{q}$ is perfect. This implies that the projection $f_{h} \in \mathcal{R}_{g}^{q}$ of $f_{h}(x)$ is the $p$-th power of some element $l \in \mathcal{R}_{g}^{q}$. Then we have

$$
l(x)^{p} \equiv f_{h}(x) \quad(\bmod g(x)),
$$

where $l(x)$ is the representative of $l$ of lowest degree and then

$$
l(x)=\sum_{i, j} c_{(i j)} \alpha^{i} x^{j} \in \mathbb{F}_{q}[x] .
$$


Hence, we can conclude that

$$
\begin{aligned}
\left(\prod_{i, j} a_{i, j, h^{\prime}}(x)^{c}(i j)\right)^{p} & =\left(1+l(x) g(x)^{h^{\prime}}+\tilde{l}(x) g(x)^{h^{\prime}+1}\right)^{p}= \\
& =1+l(x)^{p} g(x)^{p h^{\prime}}+\bar{l}(x) g(x)^{p\left(h^{\prime}+1\right)} \equiv f(x) \quad\left(\bmod g(x)^{h+1}\right),
\end{aligned}
$$

and the proof is complete.

Proof. [Theorem 21] The polynomial $f_{1}(x)$ can be expressed as

$$
f_{1}(x)=\sum_{i, j} c_{(i j, 1)} \alpha^{i} x^{j}
$$

From the previous lemma we have

$$
\prod_{i, j} a_{i, j, 1}(x)^{c}(i j, 1)=1+f_{1}(x) g(x)+m(x) g(x)^{2} .
$$

Let now consider $\tilde{f}_{2}(x) \equiv f_{2}(x)-m(x)(\bmod g(x))$; then

$$
\tilde{f}_{2}(x)=\sum_{i, j} c_{(i j, 2)} \alpha^{i} x^{j}
$$

Using the lemma once more, we obtain

$$
\prod_{i, j} a_{i, j, 2}(x)^{c_{(i j, 2)}}=1+\tilde{f}_{2}(x) g(x)^{2}+\tilde{m}(x) g(x)^{4} .
$$

Let us now multiply the relations (5) and (6):

$$
\begin{aligned}
& \left(1+f_{1}(x) g(x)+m(x) g(x)^{2}\right)\left(1+\tilde{f}_{2}(x) g(x)^{2}+\tilde{m}(x) g(x)^{4}\right)= \\
& =1+f_{1}(x) g(x)+f_{2}(x)+\hat{m}(x) g(x)^{3} .
\end{aligned}
$$

The claim is obtained by iterating this computation $l$ times.

Let us now consider the homomorphism of groups

$$
\mu: \prod_{i, j, k}\left(a_{i, j, k}(x)\right) \rightarrow S_{p}
$$

where the parameters satisfy the conditions given in (4) and in addition $p \nmid k$. To prove that the map above is an isomorphism it is enough to prove injectivity.

Theorem 23. With the conditions previously given on the parameters $i, j, k$, the following holds:

$$
\prod_{i, j, k} a_{i, j, k}(x)^{c}(i j k) \equiv 1 \quad\left(\bmod g(x)^{l}\right) \Longleftrightarrow c_{(i j k)} \equiv 0 \quad\left(\bmod \operatorname{ord}\left(a_{i, j, k}(x)\right)\right) .
$$

Proof. Let us begin by expanding the power of each polynomial $a_{i, j, k}(x)$. Writing the exponents as

$$
c_{i, j, k}=p^{s(i j k)} c_{(i j k)}^{\prime}
$$


it follows that

$$
\begin{aligned}
a_{i, j, k}(x)^{c_{(i j k)}} & =\left(1+\alpha^{i} x^{j} g(x)^{k}\right)^{c_{(i j k)}} \\
& =\left(1+\alpha^{i p^{s}(i j k)} x^{j p^{s}(i j k)} g(x)^{k p^{s}(i j k)}\right)^{c^{\prime}(i j k)} \\
& =1+\sum_{h=1}^{c_{(i j k)}^{\prime}}\left(\begin{array}{c}
c_{(i j k)}^{\prime} \\
h
\end{array}\right)\left(\alpha^{i p^{s(i j k)}} x^{j p^{s}(i j k)} g(x)^{k p^{s}(i j k)}\right)^{h} .
\end{aligned}
$$

The next step is to find the minimum exponent of $g(x)$ in $(7)$. We have to highlight all the terms where this exponent occurs to continue with the proof. Let us introduce the notation

$$
\mathfrak{K} p^{\mathfrak{s}}:=\min _{i, j, k} k p^{s(i j k)} .
$$

In order to use this notation it is important that $p \nmid k$, so that any triplet $(\bar{i}, \bar{j}, \bar{k})$ giving this minimum is such that $s_{(\bar{i} \bar{j} \bar{k})}=\mathfrak{s}$.

Now we will deal with the product $\prod_{i, j, k} a_{i, j, k}(x)^{c(i j k)}$. If we use (7) and group all monomials according to the power of $g(x)$ they contain, we obtain

$$
m_{\mathfrak{K} p^{\mathfrak{s}}}(x):=\sum_{(i, j) \in \mathcal{J}} c_{(i j k)}^{\prime} \alpha^{i p^{\mathfrak{s}}} x^{j p^{\mathfrak{s}}}=\left(\sum_{(i, j) \in \mathcal{J}} c_{(i j k)}^{\prime} \alpha^{i} x^{j}\right)^{p^{\mathfrak{s}}}
$$

where $\mathcal{J}$ is the set of all pairs $(i, j)$ for which the exponent $k p^{s(i j k)}$ is minimal.

The polynomial $m^{\prime}(x):=\sum_{(i, j) \in \mathcal{J}} c_{(i j k)}^{\prime} \alpha^{i} x^{j}$ does not vanish, because the pairs $(i, j)$ appear once in the sum. Moreover, the condition $j<\operatorname{deg} g(x)$ says that no factor of $g(x)$ divides $m^{\prime}(x)$. The hypothesis is then satisfied if and only if $\mathfrak{K} p^{\mathfrak{s}} \geq l$.

This last remark concludes the proof, because for any single factor of the product it follows that

$$
a_{i, j, k}(x)^{c}(i j k) \equiv 1 \quad\left(\bmod g(x)^{l}\right),
$$

so that $c_{(i j k)} \equiv 0\left(\bmod \operatorname{ord}\left(a_{i, j, k}(x)\right)\right)$.

5.2. Set of generators of the $x$-orbits of $M_{g^{l}}^{q}$. In this subsection we will make the representatives of the $x$-orbits of the ring explicit. We will use the results of previous sections to express these representatives via generators of the groups $M_{g^{l}}^{q}$ with $l<t$. In Corollary 17 we saw how to decompose the ring into a disjoint union of sets stable under $x$-multiplication. The representatives are then to be looked for in these sets.

Let us give the order of the generators of the $p$-Sylow subgroup of $M_{g^{l}}^{q}$.

Theorem 24. Let $l \in \mathbb{N}$. The elements $a_{i, j, k}(x) \in M_{g^{l}}^{q}$, with parameters satisfying (4) and such that $p \nmid k$, have order

$$
\operatorname{ord}\left(a_{i, j, k}(x)\right)=p^{\left\lceil\log _{p} l / k\right\rceil} .
$$

Proof. Thanks to Theorem 20, the order of the elements $a_{i, j, k}(x)$ is a power of $p$. For $m \in \mathbb{N}$ we have

$$
\left.a_{i, j, k}(x)^{p^{m}}=\left(1+\alpha^{i} x^{j} g(x)^{k}\right)^{p^{m}} \equiv 1+\left(\alpha^{i} x^{j} g(x)^{k}\right)\right)^{p^{m}} \quad\left(\bmod g(x)^{l}\right) .
$$


Since $\operatorname{gcd}\left(\alpha^{i} x^{j}, g(x)\right)=1$ we get

$$
\begin{aligned}
\left(\alpha^{i} x^{j} g(x)^{k}\right)^{p^{m}} \equiv 0 \quad\left(\bmod g(x)^{l}\right) & \Longleftrightarrow g(x)^{k p^{m}} \equiv 0 \quad\left(\bmod g(x)^{l}\right) \\
& \Longleftrightarrow k p^{m} \geq l .
\end{aligned}
$$

It follows that the lowest exponent of $p$ which fulfills the previous relation is

and the proof is complete.

$$
m=\left\lceil\log _{p} l / k\right\rceil
$$

To investigate the construction of the $x$-orbits more deeply, we have to divide the cyclic group (x) into product of others cyclic groups.

Theorem 25. Let $l \in \mathbb{N}$. The order of the element $x \in M_{g^{l}}^{q}$ is

$$
\operatorname{ord}(x)=\operatorname{ord}(g(x)) \cdot p^{\left\lceil\log _{p} l\right\rceil} .
$$

Proof. $\operatorname{ord}(x)$ is such that

$$
x^{\text {ord }(x)} \equiv 1 \quad\left(\bmod g(x)^{l}\right) \quad \Rightarrow \quad g(x)^{l} \mid x^{\operatorname{ord}(x)}-1 .
$$

If we write $\operatorname{ord}(x)=m=p^{s} \bar{m}$, with $p \nmid \bar{m}$, then

$$
x^{m}-1=x^{p^{s} \bar{m}}-1=\left(x^{\bar{m}}-1\right)^{p^{s}} .
$$

We are working in characteristic $p$, hence every irreducible factor of $x^{m}-1$ has multiplicity $p^{s}$. Relation (8) tells us that the multiplicity has to be at least $l$, then $p^{s} \geq l$, i.e. $s \geq\left\lceil\log _{p} l\right\rceil$.

We also know that

$$
g(x)\left|x^{m}-1 \Longleftrightarrow \operatorname{ord}(g(x))\right| m .
$$

The order of $x$ is the least common multiple of $p^{\left\lceil\log _{p} l\right\rceil}$ and $\operatorname{ord}(g(x))$; as these are coprime, and the claim follows.

Corollary 26. The cyclic group $(\mathrm{x}) \subset M_{g^{l}}^{q}$ is isomorphic to the product of two cyclic groups whose orders are $p^{\left\lceil\log _{p} l\right\rceil}$ and $\operatorname{ord}(g(x))$, respectively.

Remark. Using the representation of the groups $M_{g^{l}}^{q}$ given in the previous section we can make the previous corollary explicit. We have

$$
(x) \approx\left(\mathrm{x}_{p}(x)\right) \times\left(\mathrm{x}_{O_{g}}(x)\right)
$$

where $\left(\mathrm{x}_{p}(x)\right) \subset S_{p}$ and $\left(\mathrm{x}_{p}(x)\right) \subset M_{g}^{q}$. Without loss of generality we can take

$$
\mathrm{x}_{p}(x):=x^{\operatorname{ord}(g(x))} \in S_{p} \text { and } \mathrm{x}_{o_{g}}(x):=h(x)^{\frac{q^{r}-1}{\operatorname{ord}(g(x))}} \in M_{g}^{q}
$$

where $h(x)$ is the generator of $M_{q}^{q}$.

Theorem 27. Let $\mathrm{x}_{p}(x) \in S_{p} \subset M_{g^{l}}^{q}$ be an element of order $p^{\left\lceil\log _{p} l\right\rceil}$. There exist parameters $0 \leq i_{0}<\delta-1$ and $0 \leq j_{0}<\operatorname{deg} g(x)$ such that

$$
S_{p} \approx\left(\mathrm{x}_{p}(x)\right) \times \prod_{\substack{i, j, k \\(i . j . k) \neq\left(i_{0}, j_{0}, 1\right)}}\left(a_{i, j, k}(x)\right),
$$

where $(i, j, k)$ satisfy (4) and $p \nmid k$. 
Proof. The element $\mathrm{x}_{p}$ belongs to the $p$-Sylow subgroup; hence, thanks to Theorem 21, it has a representation via polynomials $a_{i, j, k}(x)$, i.e.

$$
\mathrm{x}_{p}(x) \equiv \prod_{i, j, k} a_{i, j, k}(x)^{c}(i j k) \quad\left(\bmod g(x)^{l}\right)
$$

The order of the element is the highest possible power of $p$. Thanks to the above representation, there exist $0 \leq i_{0}<\delta-1$ and $0 \leq j_{0}<\operatorname{deg} g(x)$ such that

$$
\operatorname{gcd}\left(c_{\left(i_{0} j_{0} 1\right)}, p\right)=1 .
$$

For every $e_{(i j k)} \in \mathbb{N}$ the following relation holds

$$
\prod_{i, j, k} a_{i, j, k}(x)^{e_{(i j k)}}=\mathrm{x}_{p}(x)^{\tilde{e}_{p}} \cdot \prod_{\substack{i, j, k \\(i, j, k) \neq\left(i_{0}, j_{0}, 1\right)}} a_{i, j, k}(x)^{\tilde{e}_{(i j k)}},
$$

with

$$
\begin{aligned}
\tilde{e}_{p} & \equiv e_{\left(i_{0} j_{0} 1\right)} \cdot\left(c_{\left(i_{0} j_{0} 1\right)}\right)^{-1}\left(\bmod p^{\left\lceil\log _{p} l\right\rceil}\right) \\
\tilde{e}_{(i j k)} & \equiv e_{(i j k)}-c_{(i j k)} \tilde{e}_{p} \quad\left(\bmod p^{\left\lceil\log _{p} l / k\right\rceil}\right) .
\end{aligned}
$$

The proof is complete.

We can now state the theorem that specifies every possible representative of the $x$-orbits of $M_{g^{l}}^{q}$.

Theorem 28. Let $g(x) \in \mathbb{F}_{q}[x]$ be a degree-r irreducible polynomial and $l \geq 2$. There exist $0 \leq i_{0}<\delta-1$ and $0 \leq j_{0}<\operatorname{deg} g(x)$ such that the set

$$
\left\{h(x)^{t} \cdot \prod_{\substack{(i, j, k) \neq\left(i_{0}, j_{0}, 1\right) \\ 0 \leq i<\delta, 0 \leq j<r \\ 1 \leq k \leq t, p \nmid k}}\left(1+\alpha^{i} x^{j} g(x)^{k}\right)^{c_{(i j k)}} \quad\left(\bmod g(x)^{l}\right)\right\},
$$

is a family of repretatives of orbits in $M_{g^{l}}^{q}$. Here, $h(x)$ is a primitive element of $\mathcal{R}_{g}^{q}, \alpha \in \mathbb{F}_{q}$ is an algebraic element of degree $\delta$ over $\mathbb{F}_{p}$, and $t$ and $c_{(i j k)}$ are such that

- $0 \leq t<\frac{q^{r}-1}{\operatorname{ord}(g(x))}$

- $0 \leq c_{(i j k)} \leq p^{\left\lceil\log _{p} l / k\right\rceil}$.

Proof. The theorem is quickly proved by rewriting

$$
u^{\prime} \in \mathfrak{C}_{u}^{\perp} \Longleftrightarrow \exists \nu \in \mathbb{N}: u^{\prime}(x) \equiv x^{\nu} u(x) \quad\left(\bmod g(x)^{l}\right)
$$

as

$$
u^{\prime} \in \mathfrak{C}_{u}^{\perp} \Longleftrightarrow \exists \nu, \mu \in \mathbb{N}: u^{\prime}(x) \equiv \mathrm{x}_{p}(x)^{\nu} \mathrm{x}_{o_{g}}(x)^{\mu} u(x) \quad\left(\bmod g(x)^{l}\right)
$$

and applying Theorem 27 . 
5.3. Backward Steps. In this part of the article we make some steps backward.

Our goal was to construct the representatives of the ring $\mathcal{R}_{g}^{q}$ with $g(x)$ any polynomial of $\mathbb{F}_{q}[x]$.

Using Theorem 28 we obtain, for every irreducible polynomial $g_{l}(x)$ in the decomposition of $g(x)$, the representatives of their $x$-orbits in $M_{g_{l}^{s}}^{q}$ for $1 \leq s<e_{l}$. Corollary 17 gives us the opportunity to compute the representatives of the ring $\mathcal{R}_{g_{l} e_{l}}^{q}$. This step is easily done: the representatives of $M_{g_{l}^{s}}^{q}$ for $1 \leq s<e_{l}$ multiplied with a well-chosen power of $g(x)$ give as result representatives of $\mathcal{R}_{g_{l} e_{l}}^{q}$.

By considering all possible irreducible polynomials of the decomposition of $g(x)$, we obtain representative of all rings $\mathcal{R}_{g_{l} e_{l}}^{q_{l}}$. The next step is to use Theorem 14, and with that we make the representative of the ring $\mathcal{R}_{g}^{q}$ explicit.

In involving, at the end, the fundamental step of the algorithm to the LRS's related to the representatives of $\mathcal{R}_{g}^{q}$, we obtain the weight distribution of the dual code of a CRC code.

\section{Conclusions}

We analyzed the complexity of the algorithm in the case of $\mathcal{R}_{g^{t}}^{q}$ where $g(x)$ is an irreducible polynomial. It turns out to be

$$
\mathcal{O}\left(p^{\delta r} \delta r t^{2}(n+p t)\right)
$$

but since $p t$ is smaller then $n$ in any practical application the complexity can be reduced to

$$
\mathcal{O}\left(p^{\delta r} \delta r t^{2} n\right)
$$

If we consider all the elements of the ring $\mathcal{R}_{g^{t}}^{q}$ instead of only some representatives, the complexity is

$$
\mathcal{O}\left(p^{\delta r t} n\right) \text {. }
$$

It is easy to see that the two complexities differ only with respect to the $t$ parameter. The complexity in our case is polynomial in $t$, while in the other case it is exponential.

Acknowledgments. The author is grateful to Patrizia Gianni and Barry M. Trager for their guidance during this work.

\section{REFERENCES}

[CBH93] Castagnoli, G.; Bräuer, S.; Hermann M.: Optimization of Cyclic RedundancyCheck Codes with 24 and 32 Parity Bits, IEEE Trans. on Communications, Vol 41, no. 6, Giugno 1993, pp. 883-892.

[Kro81] Kronecker, L.; Zur Theorie der Elimination einer Variabeln aus zwei algebraischen Gleichungen. Monatsberichte der Königlich PreussischenAkademie der Wissenschaften, pp. 535-600, 1881.

[LN93] Lidl, R.; Niederreiter, H.: Finite Fields, Encyclopedia of Mathematics and its Application, Gian-Carlo Rota, 1993.

[MS88] MacWilliams, F. J.; Sloane, N. J. A.: The Theory of Error-Correcting Codes, North Holland, 1988.

[Ros01] Rosenthal, J.J.; Connection Between Linear Systems and Convolutional Codes, 2001. 
[Wic95] Wicker, S.B.; Error Control Systems for Digital Communication and Storage, Practice Hall, 1995.

Mathematics Institute, Winterthurerstr. 190, CH - 8057 ZüriCH

E-mail address: felice.manganiello@math.unizh.ch 